\title{
Das Internet der Dinge unter dem Aspekt der Selbststeuerung - ein Überblick
}

\author{
DIPL.-KFM. JOCHEN SCHNEIDER \\ DiPL.-KFF. AYNUR ARSLAN \\ FACHGEBIET LOGISTIK (FLOG) DER FAKULTÄT MASCHINENBAU, UNIVERSITÄT DORTMUND
}

\section{Zusammenfassung}

In den letzten Jahren wurde die Vision einer Welt smarter Alltagsgegenstände unter den Begriffen wie Ubiquitous Computing, Pervasive Computing und Ambient Intelligence in der Öffentlichkeit wahrgenommen. Die smarten Gegenstände sollen mit digitaler Logik, Sensorik und der Möglichkeit zur Vernetzung ausgestattet werden. Somit bilden sie ein „Internet der Dinge“, in dem der Computer als eigenständiges Gerät verschwindet und in den Objekten der physischen Welt aufgeht.

Während auf der einen Seite die Vision des „Internet der Dinge“ durch die weiter anhaltenden Fortschritte in der Informatik, Mikroelektronik, Kommunikationstechnik und Materialwissenschaft zumindest aus technischer Sicht wahrscheinlich mittelfristig realisiert werden kann, müssen auf der anderen Seite die damit zusammenhängenden ökonomischen, rechtlichen und sozialen Fragen geklärt werden. Zur Weiterentwicklung und Realisierung der Vision des „Internet der Dinge“ wurde erstmals vom AutoID-Center das EPC-Konzept entwickelt, welches auf globale netzbasierte Informationsstandards setzt und heute von EPCglobal weiterentwickelt und umgesetzt wird. Der EPC erlaubt es, umfassende Produktinformationen über das Internet zur Verfügung zu stellen.

Die RFID-Technologie stellt dabei die wichtigste Grundlage des „Internet der Dinge“ dar, da sie die Brücke zwischen der physischen Welt der Produkte und der virtuellen Welt der digitalen Daten schlägt. Die Objekte, die mit RFID-Transpondern ausgestattet sind, können miteinander kommunizieren und beispielsweise ihren Weg durch die Prozesskette finden. So können sie dann mit Hilfe der auf den RFIDTranspondern gespeicherten Informationen Förderanlagen oder sonstige Maschinen ohne menschliches Eingreifen selbstständig steuern.

\section{Abstract}

Terms and Phrases like „Ubiquitous Computing“, „Pervasive Computing“ or „Ambient Intelligence“ are increasingly used in the respective literature, and it is the vision that smart objects are created and equipped with sensors and digital logic, building the "Internet of Things".

This article discusses the concept behind it and takes a look at the EPCglobal network, which is designed in order to process the data that is generated by the automatic identification systems. Based on the assumption of an "Internet of Things", products are expected to be able to route themselves through production and transport processes.

This represents a new paradigm in the field of logistics.

\section{Die Vision vom Internet der Dinge}

Unter Begriffen wie Ubiquitous Computing, Pervasive Computing, Ambient Intelligence und Silent Commerce hat sich in den letzen Jahren ein neues Paradigma von Informations- und Kommunikationstechniken entwickelt. Allen Begriffen gemeinsam ist die Vision einer Welt smarter Alltagsgegenstände. Sie sind mit digitaler Logik, Sensorik und der Möglichkeit zur Vernetzung ausgestattet und bilden somit ein Internet der Dinge, in dem der Computer als eigenständiges Gerät verschwindet und in den Objekten der physischen Welt aufgeht [Fleisch2005]. Doch was bedeutet nun Ubiquitous und Pervasive Computing? 


\section{Ubiquitous Computing}

Der Begriff des Ubiquitous Computing wurde erstmals 1991 von Marc Weiser in seinem Artikel „The Computer for the 21st Century“ beschrieben. Weiser prägte darin den allgegenwärtigen Computer, der dem Menschen im Alltag und Beruf unsichtbar und unaufdringlich im Hintergrund dient und ihn bei seinen Arbeiten und Tätigkeiten unterstützt bzw. ihn von lästigen Routineaufgaben weitestgehend befreit. Nach Weisers Auffassung basiert das Ubiquitous Computing (kurz: UbiComp) auf dem Prinzip, dass der Computer aus dem Bewusstsein ihrer Benutzer als Gerät verschwinden soll und in die alltägliche Umgebung eingewoben wird [Weiser1993].

Computer verschmelzen mit der physischen Welt über das Internet der Dinge und verschwinden aus der Sicht der Anwender als einzelner, identifizierbarer Gegenstand. So werden Objekte des täglichen Lebens mit informationstechnischen Teilen kombiniert und zu hybriden Objekten bzw. smarten Dingen. Aus diesem Grund soll die Rolle der Informationstechnik in den Hintergrund treten, damit sich die Menschen voll und ganz auf die Bewältigung ihrer Aufgaben und ihres Lebens konzentrieren können, weil seitens des Anwenders keine gesonderte Aufmerksamkeit mehr erforderlich ist [Paff2002]; [Leimeister2002].

\section{Pervasive Computing}

Der Begriff Pervasive Computing ist letztlich als Synonym für Ubiquitous Computing anzusehen. Pervasive Computing, welches von der Industrie geprägt wird, versucht, die überall eindringende und allgegenwärtige Informationsverarbeitung kurzfristig im Rahmen von Electronic-Commerce-Szenarien und Web-basierten Geschäftsprozessen nutzbar zu machen.

Die Perspektiven des Pervasive Computing wurden von IBM-Chairman Lou Gerstner einmal so beschrieben: „A billion people interacting with a million ebusinesses through a trillion interconnected intelligent devices" [Mattern2003]. Ein weiterer, häufig verwendeter Begriff ist das Ambient Computing (zu Deutsch etwa: Umgebungsintelligenz). Ambient Computing ist ein von der Europäischen Union geprägtes technologisches Paradigma, das ebenfalls auf die Durchdringung der Welt mit Computern und der Ausstattung alltäglicher Gegenstände mit Intelligenz sowie der Vernetzung aller Dinge abzielt [Leimeister2002]; [Böhmann2004].

Grundsätzlich bezeichnen alle Begriffe jedoch das gleiche technische und ökonomische Konzept. Die automatische Identifikation spielt bei UbiComp-Anwendungen eine wichtige Rolle. Weil passive Gegenstände im Unterschied zu Geräten selbst keine Aktionen ausführen können oder sollen, benötigen sie die Fähigkeit zur automatischen Identifikation. Die UbiComp-Infrastruktur kann mittels eines virtuellen Stellvertreters („,virtual counterpart“) des Gegenstands die Dienste Lokalisierung, Zustandsüberwachung und die Informationsübermittlung erbringen. Daher verlagern UbiComp-Anwendungen Aktivitäten aus der realen Welt in die virtuelle Welt und ersetzen Mensch-zu-Maschine- durch Maschine-zu-Maschine- Interaktionen [Strassner2005].

\section{Das EPCglobal Netzwerk}

Für die Kommerzialisierung und weitere Entwicklung der durch das AutoID-Center entwickelten Normen und Standards wurde 2003 EPCglobal als Joint Venture des Uniform Code Council (UCC) und EAN International gegründet. Um eine branchenunabhängige Nutzung der RFID-Technologie in den unternehmensübergreifenden Prozessen der Wertschöpfungskette zu ermöglichen, entwickelt EPCglobal weltweit gültige Standards [Vogel2005]. Für jedes eigenständige Thema gibt es innerhalb von EPCglobal eine Arbeitsgruppe, in der Unternehmen gemeinsame Lösungen erarbeiten. Dabei betrifft dies sowohl Anwenderanforderungen als auch die technische Umsetzung. Auf diese Weise möchte die Organisation sicherstellen, dass die EPC-Spezifikationen den Bedürfnissen der Anwender entsprechen und somit gute Aussichten haben, durch eine schnelle Umsetzung zum Standard zu werden.

Der wohl wichtigste Bestandteil des EPCglobal-Netzwerks ist der Electronic Product Code (EPC), der auf den RFID-Transpondern gespeichert ist und damit eine eindeutige Identifikation einzelner Transponder ermöglicht. Die Grundidee des EPCglobal-Netzwerks ist, die Produktinformationen mit Hilfe des Internets jederzeit verfügbar zu machen. Durch die Nutzung des Internets und die Errichtung zentraler Informationsverzeichnisse lassen sich Informationsanfragen schnell und gezielt beantworten. 
Wenn z. B. ein Supermarkt die Korrektheit der Lieferung prüfen und die weitere Handhabung sicherstellen will, kann das Unternehmen den EPC der Palette an das EPCglobal-Netzwerk senden. Das EPCglobal-Netzwerk greift auf die Homepage des Objektes zu und gibt die passenden Lieferscheininformationen zurück [Gillert2007]]. Darüber hinaus können bspw. die Bestände von weltweit verteilten Konsignationslagern in kurzen Zeitabständen überwacht werden, welches im Rahmen des klassischen Informationsaustauschs mittels EDI-Nachrichten nur eingeschränkt möglich ist. Zudem können durch die verbesserte Überwachung der Lagerbestände Einsparungen aufgrund der kürzeren Bestellzyklen und der Verringerung der Bestände erzielt werden.

Doch neben all den genannten Vorteilen gibt es auch einige Nachteile des EPC-Konzepts, einmal abgesehen von den vielfältigen physikalischen Faktoren, die zu berücksichtigen sind. Insbesondere die ständige Abrufbarkeit von Daten aus zentralen Datenbanken führt bei Daten- und Verbraucherschützern regelmäßig zu Irritationen, und polemische Berichterstattungen in den Medien, speziell in den Pioniertagen von RFID, haben die Endverbraucher stark verunsichert. Bei der zurückliegenden Fußball-WM in Deutschland, bei der erstmalig RFID-Eintrittskarten eingesetzt wurden, waren viele Besucher völlig überrascht, wenn ihnen versichert wurde, dass man sie im Stadion nicht orten kann. Aber auch ökonomisch wird das Konzept des EPC kritisiert, weil die Unternehmen, die einen EAN Nummernkreis nutzen, dafür bereits jährliche Gebühren abführen und nur selten dazu bereit sind, für den gleichen Kreis als EPC noch einmal zu zahlen.

\section{Das Konzept der Selbststeuerung}

UbiComp-Technologien verlängern das Internet über die rein digitale Welt der Computer hinaus in die physische Welt der Dinge. Im Internet der Dinge fängt die Welt der Gegenstände selbstständig an, mit den Planungs- und Überwachungssystemen zu kommunizieren, sich auszuweisen und über den eigenen Status zu informieren. Dazu kann auf den RFID-Transpondern ein Teil der logistischen Steuerungsfunktionen gespeichert werden, so dass einzelne Objekte eigenständig ihren Weg finden. Außerdem können sie durch Software auf der Basis multipler Agententechnologie autonom die für ihre Weiterbearbeitung benötigten Ressourcen anfordern oder Lösungen in Engpasssituationen finden [Bretzke2005].

Unter dem Begriff „Selbststeuerung“ versteht man die dezentrale Steuerung autonomer logistischer Objekte in einer heterarchischen Organisationsstruktur [Voigt2006]. Das bedeutet, dass die einzelnen logistischen Objekte wie das Transportgut, die Ladungsträger und auch die Transportsysteme durch neue Informations- und Kommunikationstechnologien selbstständig Steuerungsentscheidungen treffen und ihren Weg von selbst durch inner- und außerbetriebliche Netze bestimmen. Die Gesamtkonzeption bezeichnet man als das Internet der Dinge. Es ermöglicht und verlangt neue Steuerungsstrategien und -systeme, die den sich immer schneller verändernden Anforderungen heutiger Märkte besser gerecht werden als die gegenwärtigen Logistiksteuerungsund -informationssysteme.

Im Internet der Dinge muss die Ware in Echtzeit identifiziert werden. Real World Awareness (RWA) bezeichnet in diesem Zusammenhang die automatische Verbindung realer physischer Objekte mit der steuernden Software [Gillert2007]]. Um dies umzusetzen, müssen zunächst auf einem Transponder die Ziele, Zwischenziele und der Zielort eines Objektes gespeichert werden. Danach werden beginnend am Wareneingang in alle intelligenten logistischen Objekte (z. B. mit Tags ausgestattete Behälter) die nächsten Zielinformationen geschrieben. Diese Informationen werden dann von einer vollständig dezentral aufgebauten Steuerungstechnik, welche die Objekte ohne eine übergelagerte Entscheidungsinstanz autonom zum Ziel steuert, an allen Entscheidungsstellen ausgelesen. Um von Störungen einer Strecke unabhängig zu sein, werden neben der einfachen Quelle-ZielBeziehung auch alternative Wege auf dem Transponder gespeichert.

Hierdurch kann der Behälter auf verschiedenen Wegen zum Ziel finden und ggf. auch alternative Transportmittel (wie etwa ein Flurförderzeug) anfordern. So kann im Idealfall eine sehr zuverlässige Materialflusssteuerung aufgebaut werden. Datenverluste an sich treten nicht auf, da die Zielinformationen fest mit dem Behälter verbunden sind und weitere Ereignisse im eingebetteten Tag gespeichert werden können. Material- und Informationsflüsse sind stets synchron. Allerdings ist der Verlust eines Transponders in diesem Szenario ein schwerwiegendes Problem, weshalb gegenwärtig große Bemühungen in eine feste Integration der Transponder in Behälter bereits zum Zeitpunkt der Herstellung investiert werden. 


\section{Fazit}

Zusammenfassend kann man sagen, dass das Internet der Dinge neue Möglichkeiten für die Visibilität und die Rückverfolgbarkeit von Waren und Informationen innerhalb komplexer Netzwerke erschließt. Die Steuerung autonomer logistischer Systeme findet ihre logistische Fortsetzung in agentenbasierter Kommunikation und Organisation. Ein selbstorganisierendes, logistisches System auf der Basis autonomer, echtzeitnaher Objekte bzw. des Internet der Dinge wird daher oft auch als Paradigmenwechsel in der Logistik bezeichnet. Es gilt nun, in enger Zusammenarbeit mit den Daten- und Verbraucherschützern den Einsatz von Transpondern auch auf der Ebene der Endkunden und Privatpersonen nutzbar zu machen, um alle Potenziale auszuschöpfen und Missbrauchsrisiken weitestgehend auszuschließen.

\section{Literatur}

[Böhmann2004]

[Bretzke2005]

[Fleisch2005]

[Gillert2007]

[Leimeister2002]

[Mattern2003]

[Paff2002]

[Strassner2005]

[Vogel2005]

[Voigt2006]

[Weiser1993]
Böhmann, T.; Junginger, M.; Leimeister, J.M. (2004): From Disappearing Computers to Disappearing Information Management? Potenziale und Herausforderungen des Ubiquitous Computing für das Informationsmanagement der Zukunft, URL: http://www.winfobase.de/lehrstuhl/publikat.nsf/intern01/ D65E6EA5495377ECC125703E003F196C/\$FILE/04-31.pdf, 25.10.2006. Bretzke, W. R. (2005): White Paper: CONSUMER, INDUSTRIAL \& TECHNOLOGY - Radio Frequency Identification (RFID), Funktionsweise, Einsatzbereiche und Nutzenpotenziale - Eine zukunftsweisenden Technologie, URL: http://www.bearingpoint.de/media/library_industry_cim/ EU_C8252_GER_White_Paper_RFID.pdf, 16.09.2006.

Fleisch; E.; Mattern, F. (2005): Vorwort, in: Internet der Dinge - Ubiquitous Computing und RFID in der Praxis: Visionen, Technologien, Anwendungen, Handlungsanleitungen, Mattern, F. (Hrsg. 2005), Berlin / Heidelberg, Springer 2005. Gillert, F.; Hansen, W. R. (2007): RFID für Optimierung von Geschäftsprozessen Prozess-Strukturen, IT-Architekturen, RFID-Infrastruktur, München, Hanser Verlag, 2007.

Leimeister, J. M.; Krcmar, H. (2002): Ubiquitous Computing, in: Das Wirtschaftsstudium, Oktober 2002, 31. Jahrgang, Nr. 10, S. 1284-1294.

Mattern, F. (2003): Vom Verschwinden des Computers - Die Vision des Ubiquitous Computing, in: Total vernetzt, Szenarien einer informatisierten Welt, Mattern, F. (Hrsg. 2003), Berlin et. al., Springer 2003, S. 1-41.

Pfaff, D.; Skiera, B. (2002): Ubiquitous Computing - Abgrenzung, Merkmale und Auswirkungen aus betriebswirtschaftlicher Sicht, URL: http://www.ecommerce.wiwi.unifrankfurt.de/pfaff/publikationen/ beitrag_liechtenstein.pdf, 17.09.2006.

Strassner, M. (2005): RFID im Supply Chain Management - Auswirkungen und Handlungsempfehlungen am Beispiel der Automobilindustrie, Diss. Univ. St. Gallen, Hochschule für Wirtschafts-, Rechts- und Sozialwissenschaften, 2005.

Vogel, K. (2005): GS1 Germany - Entwicklungsstand Management-Information, Zum Stand der RFID/EPC-Standardisierung und -Implementierung, URL: http://www.gs1-germany.de/content/e39/e466/e468/datei/epc_rfid/ mip_2_entwicklungsstand_gs1.pdf, 20.09.2006.

Voigt, K. I.; Saatman, M.; Schorr, S. (2006): Einsatzfelder und Grenzen der Selbststeuerung in der Logistik, in: Wissenschaft und Praxis im Dialog, Steuerung von Logistiksystemen - auf dem Weg zur Selbststeuerung, Pfohl, H. C. / Wimmer, T. (Hrsg. 2006), Hamburg, Deutscher Verkehrs-Verlag 2006, S. 96-115. Weiser, M. (1993): Some Computer Science Issues in Ubiquitous Computing, URL: http://www.ubiq.com/hypertext/weiser/UbiCACM.html, 25.09.2006. 